\title{
Multi-Parameter Based Performance Evaluation Of Classification Algorithms
}

\author{
Saurabh Kr. Srivastava ${ }^{1}$ and Sandeep Kr. Singh ${ }^{2}$ \\ ${ }^{1}$ Research Scholar, Department of Computer Sc. \& Engineering, JIIT University, Noida, \\ India \\ ${ }^{2}$ Assistant Professor, Department of Computer Sc. \& Engineering, JIIT University, Noida, \\ India
}

\begin{abstract}
Diabetes disease is amongst the most common disease in India. It affects patient's health and also leads to other chronic diseases. Prediction of diabetes plays a significant role in saving of life and cost. Predicting diabetes in human body is a challenging task because it depends on several factors. Few studies have reported the performance of classification algorithms in terms of accuracy. Results in these studies are difficult and complex to understand by medical practitioner and also lack in terms of visual aids as they are presented in pure text format. This reported survey uses ROC and PRC graphical measures to improve understanding of results. A detailed parameter wise discussion of comparison is also presented which lacks in other reported surveys. Execution time, Accuracy, TP Rate, FP Rate, Precision, Recall, F Measure parameters are used for comparative analysis and Confusion Matrix is prepared for quick review of each algorithm. Ten fold cross validation method is used for estimation of prediction model. Different sets of classification algorithms are analyzed on diabetes dataset acquired from UCI repository.
\end{abstract}

\section{KEYWORDS}

Data mining, Diabetes, UCI repository, Machine Learning.

\section{INTRODUCTION AND LITERATURE REVIEW}

Diabetes invites other chronic diseases. It is primarily associated with an increase of blood glucose. Two common categories of diabetes are Type-1 and Type-2. Type-1 diabetes occurs when pancreas fails to produce sufficient insulin while Type- 2 occurs when body cannot effectively consume the insulin produced. Diabetes confirmation requires a lot of examination that affects time and cost both. Prior studies show that classification algorithms have been effectively used in prediction of diabetes.

Several studies and results are reported using data mining techniques in healthcare for classification in medical databases. In the context of that J.W.Smith et al.[1] have introduced adaptive learning routine that executes digital analogy of perceptions called ADAP. The algorithm uses 576 training instances and classification accuracy is $76 \%$ on the remaining 192 instances. K.Srinivas et al.[4] studied the applications of Data mining Techniques in healthcare and have reported prediction of heart attacks using clinical database. Elmakolce et al.[5] has given the glimps of different data mining techniques utilization. Asha Rajkumar et al.[2] and A.Khemphlila et al.[3] discussed various data mining techniques for diagnosis of certain life threatening diseases. HuyNguyen A.P. et al.[6] proposed a new Homogeneity-Based Algorithm that determines over fitting and over generalization behavior of classification. Recently K.R. Lakshmi et al.[10] and Karthikeyini.V. et.al [8,9] discussed data mining algorithms performance based upon their computing time, and precision value. 
The data mining techniques aims to extract the hidden information from large dataset and transform it into knowledge patterns for further use that can be easily understood by human. A model is an example of structure which is based upon predicted data. The paper uses ROC and PRC graphical measures to visualize the results that makes data easy to understand by medical practitioners. Execution time, Accuracy, TP Rate, FP Rate, Precision, Recall, and F Measure parameters are used for comparative analysis.

We have described the results of experiments in which categorical (Tree, Function, Rule \& Bayesian) algorithms are used for performance evauation. The paper is organized as follows: in section 2 we described the algorithms which are used for performance evaluation; in section 3 we discussed the measures on which result is obtained; in section 4 we explained about the experimental setup, data set and result evaluation process; in section $5 \& 6$ we described the result and discussion on evaluated results; finally, in section 7 we presented our conclusion.

\section{ALGORITHMS USED FOR RESULT EVALUATION}

\subsection{Decision Tree Based Classification}

Decision Tree maps observation to conclusion in the form of target values. Decision tree separates input space of a data set into mutually exclusive regions. Each of which is assigned to a label, a value or an action to characterize its data points.Two variants: J48 and RandomTree are discussed in subsections.

2.1.1. J48: A decision tree is a graphical representation which consist of internal and external nodes connected by branches. Internal node is responsible for implementing decision functions, that determines which node to visit next. The external node, has no child node and it is associated with a value that characterizes the given data which leads to its being visited. Decision tree construction algorithms involve a two-step process. First- growing, Second-pruning. The growing process sets up a very large decision tree and pruning process reduces the large size and overfiting of the data. The pruned decision tree that is used for classification is called as classification tree [19]. A J48 decision tree is a predictive machine-learning model that decides the target value (dependent variable) of a new sample based on various attribute values of the available data. The nodes of a J48 decision tree denote the different attributes. Branches between the nodes tell us the possible values that these attributes can have in the observed samples. To build a decision tree, we need to calculate the entropy and information gain [18].

Entropy: $\mathrm{E}(\mathrm{S})=\sum$-pi $\log 2 \mathrm{pi}$ (Where $\sum$ varies from $\mathrm{i}=1$ to $\left.\mathrm{c}\right)$ Information Gain: Gain $(T, X)=$ Entropy $(T)-$ Entropy $(T, X)$.

2.1.2. Random Tree: It is the simplest tree algorithm that comes as a result of stochastic process. Random binary tree refers system model that selects random values over time. Two different distributions are commonly used for Random Tree formation: 1) Inserting nodes one at a time according to a random permutation. 2) Chosing from a uniform discrete distribution. Repeated splitting is another distribution to form the random tree. Adding and removing nodes directly in a random binary tree will generaly disrupt its random structure. In order to balance the nodes of Random Trees, random permutation are used for dynamic insertion and deletion of nodes.

\subsection{Function Based Classification}

It's a mathematical classification (or statistical procedure) to classify an instance in a particular class. 
2.2.1. Logistics Regression: Logistics Regression predicts the probability of an outcome that can only have two values. It performs a least-square fit of a parameter vector $\beta$ to a numeric target variable $\mathrm{X}$. The logistic regression uses equation: $F(X)=\beta T$. $X$ to formulate prediction model. Where $X$ is the input vector (a constant term to accommodate the intercept), and $\beta$ is parameter vector to a numeric target variable. It is possible to use this technique for classification by directly fitting logistics regression models to class indication variables. The prediction is based on the use of one or several predictors (numerical and categorical). A linear regression is not appropriate for predicting the value of binary variable for two reasons:

1. A linear regression will predict values outside the acceptable range (e.g. predicting probabilities outside the range 0 to 1 )

2. Since the two value experiments can only have one of two possible values for each experiment, the residuals will not be normally distributed about the predicted line.

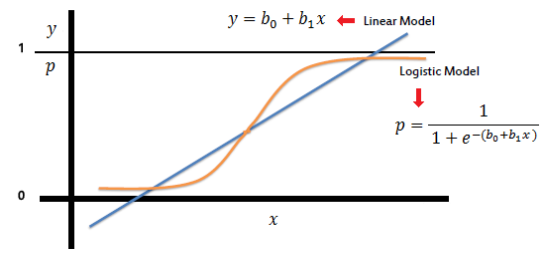

Figure 1. Logistics Regression Function

Simply, the logistic regression equation can be written in terms of an odds ratio-

$$
\mathrm{p} / 1-\mathrm{p}=\exp \left(\mathrm{b}_{0}+\mathrm{b}_{1} \mathrm{x}\right) \text {. }
$$

Here, $b_{0}$ is the constant responsible for moving the curve left and right while $b_{1}$ defines the steepness of the curve.

2.2.2. Multi-Layer Perceptron: A simplest form of neural network needs to classify linearly seperable patterns. While for non-linear patterns multi-layer perceptron (MLP) neural network model performs well. It maps sets of input data onto a set of appropriate outputs. MLP consists of multiple layers of nodes in a directed graph with each layer fully connected to the next one. Except for the input nodes, each node is a neuron (or processing element) with a nonlinear activation function. MLP uses back propagation learning algorithm for training and widely used in pattern classification and recognition. The simplest form of MLP is shown as-

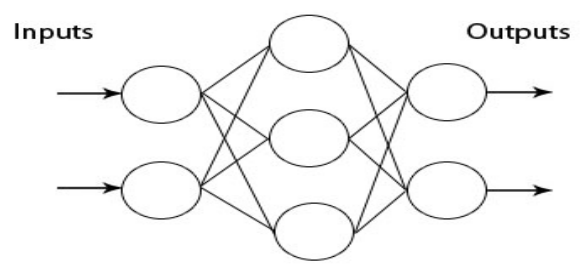

Figure 2. Multi-Layer Perceptron

\subsection{Rule Based Classification}

The rule based classification is based upon rules like (if-else). Rules are mutually exclusive and exhaustive.Two variants are discussed in following subsections. 
2.3.1. ZeroR: ZeroR is a simplest method which only focuses on the class. It is a least accurate classifier only predicts the majority category (class). It is useful for determining a baseline as a benchmark for other classification methods. ZeroR algorithm ignores the predictors only constructs a frequency table for the target and select its most frequent value.

2.3.2. OneR: OneR is a rule based classification that uses a single predictor. It generates one rule for each predictor in the data and constructs frequency table for each predictor against the target. OneR short for "One Rule" is an accurate classification algorithm, for each value of predictor, OneR makes rule as follows-

1: Count how often each value of target (class) appears

2: Find the most frequent class

3: Make the rule assign that class to this value of predictors

4: Calculate the total error of the rules of each predictor

5: Choose the predictor with smallest total error.

OneR produces rules only slightly less accurate than state-of-the-art classification algorithms.

\subsection{Bayesian Based Classification}

Bayesian classification is based upon Baye's probability rules and depends on likelihood functions. Two variants are discussed in following subsections.

2.4.1. Simple Baye's Classification: Bayesian networks are a powerful probabilistic representation for classification. The Baye's provides a rule for calculating posterior probability. If probability of item $\mathrm{A}$ is to be calculated over given item B then according to Baye's-

$\mathrm{P}(\mathrm{A} / \mathrm{B})=\mathrm{P}(\mathrm{B} / \mathrm{A}) * \mathrm{P}(\mathrm{A}) / \mathrm{P}(\mathrm{B})$

$P(A / B)$ is probability of $A$ given that $B$ is true (posterior probability).

$\mathrm{P}(\mathrm{B} / \mathrm{A})$ is probability of $\mathrm{B}$ given that $\mathrm{A}$ is true (likelihood).

$\mathrm{P}(\mathrm{A})$ is prior probability of the class.

$\mathrm{P}(\mathrm{B})$ is prior probability of predictor.

2.4.2. Naive Baye's: The Naive Baye's classifier is based on Baye's Theorem with independent assumptions between predictors. Naive Bayesian model is easy to build without complicated iterative parameter estimation. It analyzes all the attributes in the data individually, means the value of a predictor $(\mathrm{X})$ on a given $(\mathrm{C})$ is independent of the values of other predictors. This assumption is called as class conditional independence. The working steps for Naive Baye's classifier are as follows-

1. First calculating the posterior probability and construct the frequency table against the target.

2. Transforming the frequency table into likelihood table and using the Naive Baye's equation to calculate the posterior probability for each class.

3. Class with highest probability is the outcome of prediction.

$\mathrm{P}(\mathrm{C} / \mathrm{X})=\mathrm{P}(\mathrm{X} / \mathrm{C}) * \mathrm{P}(\mathrm{C}) / \mathrm{P}(\mathrm{X})$

$\mathrm{P}(\mathrm{C} / \mathrm{X})$ is posterior probability of class (target) given predictor (attribute).

$\mathrm{P}(\mathrm{X} / \mathrm{C})$ is likelihood which is the probability of predictor given class.

$\mathrm{P}(\mathrm{C})$ is prior probability of the class.

$\mathrm{P}(\mathrm{X})$ is prior probability of predictor. 
International Journal of Computer Science \& Information Technology (IJCSIT) Vol 7, No 3, June 2015

\section{MEASURES ON WHICH RESULT IS OBTAINED}

3.1. Execution Time: The time taken by the tool to execute and evaluate an algorithm.

3.2. Accuracy: Accuracy defines percentage of correctly classified instances from the test set by the classifier.

3.3. TP Rate: True Positive rate is proportion of examples which were classified as a class $X$ among all examples which truly have the X class. This shows how much the part of the class is truly captured.

3.4. FP Rate: False Positive is the proportion of the examples which were incorrectly identified and belong to another class.

3.5. Precision: Precision is the proportion of examples which truly have class $X$ among all those which were classified as X. It is a measure of exactness. Positive predictive value is called as precision.

$$
\mathrm{PPV}=\mathrm{TP} / \mathrm{TP}+\mathrm{FP}
$$

3.6. Recall: Recall is the proportion of examples which were classified as class $X$ among all examples which truely have class X. It is a measure of completeness. Negative predictive value is called as recall.

$$
\mathrm{NPV}=\mathrm{TP} / \mathrm{TP}+\mathrm{FN}
$$

3.7. F Measure: $\mathrm{F}$ measure is aggregate of precision and recall, The formula is defined as: $2 *$ Precision * Recall / Precision + Recall.

3.8. ROC Area: ROC is comparison of two operating characteristics TPR and FPR. It is also known as a receivers operating characteristic curve. A receiver operating characteristic curve is a graphical measure which interprets the performance of a classifier as its discrimination threshold is varied. It is an outcome of plotting the true positive rate vs. false positive rate at various threshold settings. True positive rate is fraction of true positives out of the total actual positives while the fraction of false positives out of the total actual negatives indcates false positive rate. The point in threshold curve record various statistics such as true positive, false positive etc. The curves are generated by sorting the prediction produced by the classifier in descending order of probability it assigns to the positive class. The formula is defind as:

ROC Area: TP Rate $=\mathrm{TP} / \mathrm{TP}+\mathrm{FN} * 100, \mathrm{FP}$ Rate $=\mathrm{FP} / \mathrm{FP}+\mathrm{TN} * 100$.

3.9. PRC Area: The PRC is known as precision recall characteristics curve. It is a comparison of two operating characteristics (PPV and sensitivity) as the criterion changes. A precision recall curve or PRC curve is a graphical plot which illustrates the performance of binary classifiers as its discrimination threshold is varied. PPV is fraction of true positives out of test outcomes positive. While sensitivity is fraction of true positive out of conditions positive.

3.10. Confusion Matrix: It is also called as contingency table. In our case the result is in two classes so it is 2 X 2 confusion matrix. Number of correctly classified instances are diagonal in the matrix and others are incorrectly classified.

\section{EXPERIMENTAL SETUP}

[20] Weka environment is a popular suit of machine learning written in java developed at the university of Waikato, New Zealand. It provides GUI to its user. Weka provides a wide range of uni and multivariate parametric and non parametric tests. It is having list of feature selection 
International Journal of Computer Science \& Information Technology (IJCSIT) Vol 7, No 3, June 2015

techniques. Weka supports data preprocessing, clustering, classifications, regression, visualization, and feature selection.

\subsection{Data Source}

To evaluate and analyze data mining classification algorithms UCI Diabetes data set is used this data set have nine attributes and 768 instances.

Table 1. Data Descriptiion

\begin{tabular}{|c|c|c|}
\hline S.No. & Name & Description \\
\hline 1. & Pregnancy & Number of times pregnant \\
\hline 2. & Plasma & Plasma glucose concentration a 2 hours in an oral glucose tolerance test. \\
\hline 3. & Pres & Diastolic blood pressure (mm Hg) \\
\hline 4. & Skin & Triceps skin fold thickness $(\mathrm{mm})$ \\
\hline 5. & Insulin & 2-Hour serum insulin $(\mathrm{mu} \mathrm{U} / \mathrm{ml})$ \\
\hline 6. & Mass & Body mass index (weight in $\mathrm{kg} /\left(\mathrm{height} \mathrm{in} \mathrm{m}^{2}\right)$ \\
\hline 7. & Pedi & Diabetes pedigree function \\
\hline 8. & Age & Age(in years) \\
\hline 9. & Class & Class variable (False or True) \\
\hline
\end{tabular}

\subsection{Result Evaluation}

Sets of algorithms (Tree, Function, Rule \& Bayesian) used for performance evaluation in terms of their Execution time, Accuracy, Precision, Recall, ROC and PRC. Followings are the steps taken up for their evaluation.

\section{Step 1}

\subsubsection{Pre-processing}

During the pre-processing the dataset which is used for evaluation is processed using filters. Basically, data pre-processing helps in removing out of range values, missing values, impossible data combinations etc. The quality of data is first and foremost requirement before analysis. The result of data preprocessing is the final training set. Preprocessing of data is done using supervised filter discrete method [12].

\section{Step 2}

\subsubsection{Training}

During the training algorithms are applied to the data so that it learns well. A training set is used along with a supervised learning method to train a knowledge dataset which can be further used 
by an artificial intelligence machine. Cross validation aims to test the model in training phase in order to limiting the problems of data overfitting and gives an insight on how the model will generalize to an independent data set.The 10 fold cross validation is used for classification of the dataset.

\section{Step 3}

\subsubsection{Testing}

The purpose of testing is to validate whether the trained data correctly classifies the given dataset or not. 10 -fold cross validation signifies that the data is divided into 10 chunks and train 10 times, treating treating a different chunk as the holdout set each time. Testing can be done on new data as well as on the part of the dataset that has not been used for training. The aim in cross-validation is to ensure that every example from the original dataset has the same chance of appearing in the training and testing set [11].

\section{RESULT}

Execution Time, TP Rate, FP Rate, Precision, Recall, F Measure, Accuracy, ROC Area, PRC Area, and Confusion Matrix are the measures used for performance evaluation.All algorithms discussed for comparison are filtered to identify the effectiveness of their higher precision and higher accuracy of classification. The overall performance evaluation table are given below.

Table 2. Performance Evaluation of classifiers

\begin{tabular}{|c|c|c|c|c|c|c|c|c|c|c|c|}
\hline & Acc. & $\mathrm{TP}$ & FP & Pre. & Recall & F Mea. & ROC & PRC & \multicolumn{3}{|c|}{ Confusion Matrix } \\
\hline \multicolumn{9}{|c|}{ TREE BASED CLASSIFICATION } & A & $\mathrm{B}$ & \\
\hline \multirow{2}{*}{$\mathrm{J} 48$} & \multirow{2}{*}{78.26} & \multirow{2}{*}{0.73} & \multirow{2}{*}{0.40} & \multirow{2}{*}{0.73} & \multirow{2}{*}{0.73} & \multirow{2}{*}{0.71} & \multirow{2}{*}{0.75} & \multirow{2}{*}{0.73} & 115 & 113 & A True \\
\hline & & & & & & & & & 52 & 448 & B False \\
\hline \multirow{2}{*}{ Random Tree } & \multirow{2}{*}{74.61} & \multirow{2}{*}{0.66} & \multirow{2}{*}{0.39} & \multirow{2}{*}{0.67} & \multirow{2}{*}{0.66} & \multirow{2}{*}{0.66} & \multirow{2}{*}{0.66} & \multirow{2}{*}{0.65} & 147 & 121 & A True \\
\hline & & & & & & & & & 140 & 360 & B False \\
\hline \multicolumn{9}{|c|}{ FUNCTION BASED CLASSIFICATION } & A & B & \\
\hline \multirow{2}{*}{ Logistics } & \multirow{2}{*}{78.65} & \multirow{2}{*}{0.75} & \multirow{2}{*}{0.33} & \multirow{2}{*}{0.75} & \multirow{2}{*}{0.75} & \multirow{2}{*}{0.75} & \multirow{2}{*}{0.81} & \multirow{2}{*}{0.80} & 155 & 113 & A True \\
\hline & & & & & & & & & 77 & 423 & B False \\
\hline \multirow{2}{*}{ MLP } & \multirow{2}{*}{75.91} & \multirow{2}{*}{0.71} & 024 & 071 & 71 & 071 & 077 & 078 & 158 & 110 & A True \\
\hline & & & 0.34 & 0.71 & $0 . / 1$ & $0 . / 1$ & 0.17 & 0.18 & 110 & 390 & B False \\
\hline & & LE B & SED & LASS & [FICA] & $\mathrm{DN}$ & & & A & B & \\
\hline One P & 7474 & 073 & 030 & 0 72 & 073 & (7) & 067 & 066 & 125 & 143 & A True \\
\hline Une $\mathrm{K}$ & 14.14 & 0.13 & 0.39 & 0.12 & 0.13 & 0.12 & 0.07 & 0.06 & 62 & 438 & B False \\
\hline Zero $\mathrm{R}$ & 6510 & 065 & 065 & 042 & 065 & 51 & 0.50 & 0.54 & 0 & 268 & A True \\
\hline Zero K & & 0.05 & 0.65 & 0.42 & 0.65 & 0.51 & 0.50 & 0.54 & 0 & 500 & B False \\
\hline & & BAYE & CLA & $\overline{\text { SSIFI }}$ & EATIO & & & & $\mathrm{A}$ & B & \\
\hline Rave's & 7787 & 075 & 030 & 075 & 075 & 075 & 083 & 082 & 175 & 93 & A True \\
\hline Baye's & 17.81 & 0.15 & & & & & & & 99 & 401 & B False \\
\hline & & & & & & & & & 172 & 96 & A True \\
\hline Naive Baye's & 77.87 & 0.75 & 0.30 & 0.75 & 0.75 & 0.75 & 0.83 & 0.82 & 93 & 407 & B False \\
\hline
\end{tabular}


International Journal of Computer Science \& Information Technology (IJCSIT) Vol 7, No 3, June 2015

\section{RESULT DISCUSSION}

\subsection{Computation Time}

This measure depicts time taken by algorithm for result evaluation. In this ZeroR and Naive Baye's classifiers performs best among all.

Table 3. Computation time of classifiers

\begin{tabular}{|c|c|c|c|c|c|c|c|c|}
\hline & J48 & $\begin{array}{c}\text { Random } \\
\text { Tree }\end{array}$ & Logistics & MLP & OneR & ZeroR & Baye's & $\begin{array}{c}\text { Naive } \\
\text { Baye's }\end{array}$ \\
\hline Com.Time & $130 \mathrm{~ms}$ & $50 \mathrm{~ms}$ & $200 \mathrm{~ms}$ & $\begin{array}{c}3950 \\
\mathrm{~ms}\end{array}$ & $20 \mathrm{~ms}$ & $\begin{array}{c}\text { Less } \\
\text { than } \\
10 \mathrm{~ms}\end{array}$ & $50 \mathrm{~ms}$ & $\begin{array}{c}\text { Less } \\
\text { than } \\
10 \mathrm{~ms}\end{array}$ \\
\hline
\end{tabular}

\subsection{Accuracy, Precision, Recall, TP \& FP}

Precision and accuracy are related terms. In statistics measuring the degree of closeness is accuracy while precision is reproducibility (the degree to which repeated measurements under unchanged conditions shows the same result). When a system contains systematic error increase in sample size, increase in precision does not increase the accuracy. Eliminating the systematic error improves accuracy but does not change precision. The evaluated results are as follows-

Table 4. Accuracy, Precision, Recall, TP \& FP values

\begin{tabular}{|c|c|c|c|c|c|c|c|c|}
\hline & J48 & $\begin{array}{c}\text { Random } \\
\text { Tree }\end{array}$ & Logistics & MLP & OneR & ZeroR & Baye's & $\begin{array}{c}\text { Naive } \\
\text { Baye's }\end{array}$ \\
\hline Accuracy & 78.26 & 74.61 & 78.65 & 75.91 & 74.74 & 65.10 & 77.87 & 77.87 \\
\hline Precision & 0.726 & 0.666 & 0.747 & 0.714 & 0.724 & 0.424 & 0.751 & 0.753 \\
\hline Recall & 0.733 & 0.66 & 0.753 & 0.714 & 0.733 & 0.651 & 0.75 & 0.754 \\
\hline TP & 0.733 & 0.66 & 0.753 & 0.714 & 0.733 & 0.651 & 0.75 & 0.754 \\
\hline FP & 0.408 & 0.392 & 0.328 & 0.344 & 0.391 & 0.651 & 0.295 & 0.298 \\
\hline
\end{tabular}

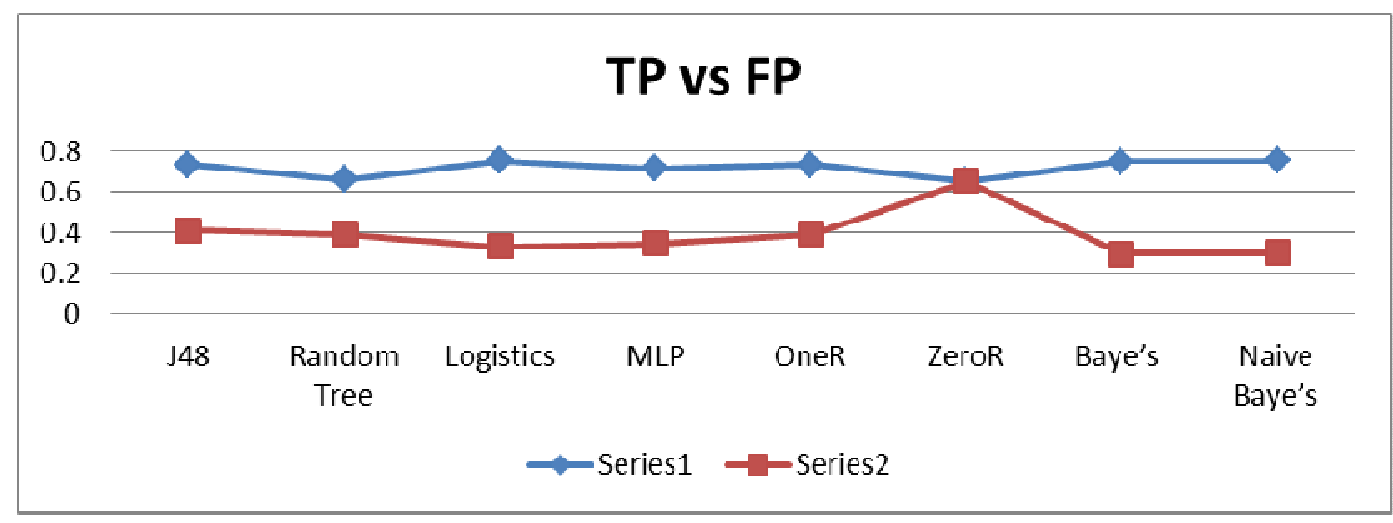

Figure 3. Simulation result of TP and FP 
In the plotted diagram (Figure 3) Series 1 represents true positive value while Series 2 represents false positive value, with the observation of the plotted data it is clearly visible that ZeroR classifier having no impact on TP and FP for this dataset.

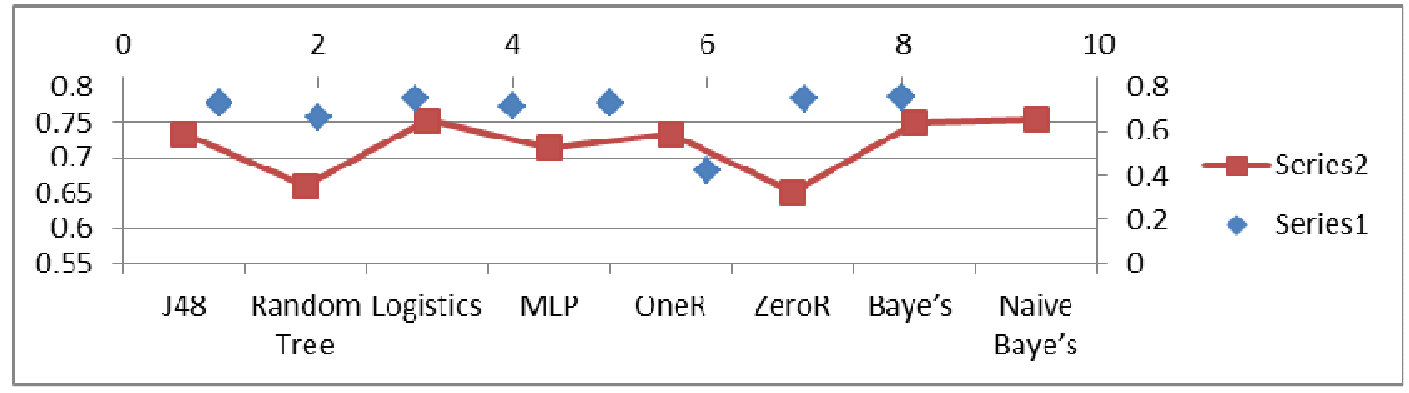

Figure 4. Simulation result of Precision and Recall

In the plotted diagram (Figure 4) Series 1 represents recall while Series 2 represents precision value.

Based on the above results, we can observe that for the diabetes dataset the highest accuracy is $78.26 \%$ (J48) and the minimum is 65.10 (ZeroR) while in case of precision Naive Baye's (75.3\%) performs best among all. A measurement system is considered valid if both (accuracy $\&$ precision) are accurate and precise. In fact, with the consideration of both the measures Naive Baye's classifier present at the top and OneR lies at the bottom of the chart. Recall is the fraction of relevant instances that are retrieved. Precision and recall both are inversely proportional to each other. Both precision and recall are based on measurement of relevance. So in most of the cases performance of a classifier is always measured in terms of both. Considering both (precision \& recall) in above table, Bayesian classifier performs best among all, followed by Funtion based and Tree based classifiers, while Rule based classifier lies at the bottom.

\subsection{F-Measure \& ROC}

F-Measure aggregates the precision and recall. The classifier's performance can be measure by considering precision \& recall both.

Table 8.

\begin{tabular}{|c|c|c|c|c|c|c|c|c|}
\hline & J48 & $\begin{array}{c}\text { Random } \\
\text { Tree }\end{array}$ & Logistics & MLP & OneR & ZeroR & Baye's & $\begin{array}{c}\text { Naive } \\
\text { Baye's }\end{array}$ \\
\hline F-Measure & 0.714 & 0.663 & 0.748 & 0.714 & 0.719 & 0.513 & 0.751 & 0.754 \\
\hline ROC & 0.745 & 0.658 & 0.811 & 0.766 & 0.671 & 0.497 & 0.825 & 0.825 \\
\hline
\end{tabular}

Based on above table 8 it is clearly visible that the performance order of classifiers are: Bayesian, Funtion based, Tree based, and Rule based. While Naive baysian (F Mea. $=0.754$ ) classifier performs best among all. 


\section{CONCLUSION}

The aim of data mining algorithm is to get best among available. In this paper different sets (Tree, Function, Rule \& Bayesian) of classification algorithms are used for performance evaluation. These algorithms are compared on the basis of execution time, TP Rate, FP Rate, Precision, Recall, Accuracy, ROC and PRC. ROC and PRC are graphical representation used for ease of understanding. Weka tool is used to evaluate and investigate the performance of four different categories of classifiers. The raw numbers are shown in the confusion matrix (Table 2), with A and $\mathrm{B}$ representing the class labels. Here in the experiment we are using 768 instances, so the percentages and raw numbers add up comes out $\mathrm{AA}+\mathrm{BB}, \mathrm{AB}+\mathrm{BA}$. The percentage of correctly classified instances is often called accuracy. We compare all the parameters and found that Naive bayes classifier performs best among all while ZeroR classifier only provides benchmark for classification. ROC and PRC graphical measures are used for quick review of all classifiers. According to the result the performance order (highest to lowest) of the algorithms are: Bayesian, Function, Tree and Rule.

\section{FUTURE WORK}

Validataion of results using other datasets is required before generalizing result findings.

\section{REFERENCES}

[1] Smith, J., W., Everthart, J.,E., Dickson, W.,C., Knowler, W.,C. and Johannes, R.,S.,Using the ADAP learning algorithm to forecast the onset of diabetes mellitus, in Proceedings of the Symposium on Computer Applications and Medical Care, IEEE Computer Society Press, 1988, pp. 261-265

[2] Asha Rajkumar,Sophia Reena.G., "Diagnosis of Heart Disease Using Data Mining Algorithm", Global Journal of Computer Sci- ence and Technology, Vol-10, 2010, pp. 38-46.

[3] A.Khemphila,V.Boojing, "Comparing Performance of logistic regression,decision tree and neural network for classifying heart disease patients", Proceeding of International conference on Com- puter Information System and Industrial Management Application, 2010, pp.193-198.

[4] K.Srinivas, B.Kavitha Rani,A.Govrdhan, Applications of Data Mining Techniques in +health care and Prediction Heart Attacks, International Journal on Computer Science and Engineering (IJCSE), vol.II, 2010, pp.250-255.

[5] Elma kolce (cela), Neki Frasheri, "A Literature Review of Data Mining Techniques used in Healthcare Databases”, ICT Innova- tions 2012 Web Proceedings-Poster Session.

[6] Huy Nguyen Anh Pham and Evangelos Triantaphyllou "Prediction of Diabetes by Employing a New Data Mining Approach Which Balances Fitting and Generalization" Department of Computer Science, 298 Coates Hall, Louisiana State University, Baton Rouge, LA 70803.

[7] Ms.S.Sapna, Dr.A.Tamilarasi "Data mining - Fuzzy Neural Ge- netic Algorithm in predicting diabetes" Department Of Com- puter Applications (MCA), K.S.R College of Engineering "BOOM 2K8", Research Journal on Computer Engineering, March 2008.

[8] Karthikeyini.V., Pervin begum.I., "Comparison a performance of data mining algorithms (CPDMA) in prediction of Diabetes Disease", International journal of Computer Science and Engineer- ing, Vol.5, No. 03, March 2013, pp. 205-210.

[9] Karthikeyini.V., Pervin begum.I., Tajuddin.K., Shahina Begum, "Comparative of data mining classification algorithm (CDMCA) Diabetes Disease Prediction", International journal of Computer Applications, Vol.60, No. 12, Dec. 2012, pp. 26-31

[10] K.R. Lakshmi and S. Prem Kumar, Utilization of Data Mining Techniques for Prediction of Diabetes Disease Survivability. IJSER, Vol. 4, Issue 6, June 2013.

[11] http://www.sussex.ac.uk/Users/christ/crs/ml/lec03a.html

[12] http:// weka.sourceforge.net/doc.dev/weka/filters/supervised/

[13] http://chem-eng.utoronto.ca/ datamining/dmc/zeror.htm

[14] http://chem-eng.utoronto.ca/ datamining/dmc/oner.htm 
International Journal of Computer Science \& Information Technology (IJCSIT) Vol 7, No 3, June 2015

[15] http://chem-eng.utoronto.ca/ datamining/dmc/naive_bayesian.htm

[16] http://chem-eng.utoronto.ca/ datamining/dmc/logistic_regression.htm

[17] http://neuroph.sourceforge.net/tutorials/MultiLayerPerceptron.html

[18] Jiwei Han and Micheline Kamber: Data Mining Concepts and Techniques, Elsevier Inc., Edition 2, 2006, ISBN no. 978-81-312-0535-8.

[19] Decision Tree at http://chemeng.utoronto.ca/ datamining/dmc/decision_tree.htm

[20] WEKA Tool at http://www.cs.waikato.ac.nz/ml/weka/

\section{Authors}

Mr. Saurabh Kumar Srivastava is a research scholar in the department of Computer Science \& Engineering at JIIT Noida, India. He obtained his M-Tech (Computer Engineering) from Shobhit University and B-Tech(C.S.) from Uttar Pradesh Technical University, Lucknow (U.P.). He has been in teaching since 7 years. During his teaching he has coordinated several Technical fests and International/National Conferences at Institute level. He has attended several seminars, workshops and conferences at various levels. His area of research includes Datamining, Machine Learning, Artificial Intelligence, and Web Technology.

Dr. Sandeep Kumar Singh is an Assistant Professor (Senior Grade) at JIIT in Noida, India. He has completed his Ph.D in (Computer Science and Engineering). He has around 13+ years' experience, which includes corporate training and teaching. His areas of interests are Software Engineering, Requirements Engineering, Software Testing, Web Application Testing,Internet and Web Technology, Object Oriented Technology, Programming Languages, Information Retrieval and Data Mining, Model based Testing and Applications of Soft computing in Software Testing and Databases. He is currently supervising 4 Ph.D's in Computer Science. He has around 28 published papers to his credit in different international journals and conferences. 